\title{
Integration of Active Fiber Composite (AFC) Sensors/Actuators into Glass/Epoxy Laminates
}

\author{
Mark M. Melnykowycz*, Xavier Kornmann, Christian Huber, Andreas J. Brunner, Michel \\ Barbezat \\ Empa - Materials Science \& Technology, \\ Ueberlandstr. 129, CH-8600 Dübendorf, Switzerland
}

\begin{abstract}
In the current study Active Fiber Composites (AFC) utilizing Lead-Zirconate-Titanate (PZT) fibers with Kapton screen printed interdigitated electrodes (IDE) were integrated into orthotropic glass fiber reinforced plastic (GFRP) laminates to investigate integration issues associated with smart structures and host laminate integrity. To aid in this goal surrogate or "Dummy" AFC (DAFC) were designed using a GFRP core and Kapton outer layers to match the longitudinal mechanical and interface properties of the AFC. These DAFC were used in place of real AFC to expedite test specimen manufacture and evaluation. This allowed efficient investigation of the impact of an integrated AFClike inclusion on laminate mechanical integrity. Two integration techniques, cutout and simple insertion were investigated using DAFC, with little difference seen between the integrity of laminates prepared using these two methods. Using this testing scheme the influence of device placement in relation to position extending away from the laminate symmetric axis was found to have an effect on laminate integrity in tensile loading. As the DAFC were placed far from the laminate symmetry axis, the ultimate tensile strength and strain of the laminates decreased in a linear manner while the Young's modulus of the laminates remained constant. Similar trends were observed with integrated AFC specimens. The performance of integrated AFC was characterized using monotonic cyclic tensile loading with increasing strain levels. A transition region was observed between strains of $0.05 \%-0.50 \%$, with a dramatic decrease in AFC sensitivity from a maximum to minimum value.
\end{abstract}

Keywords: Active Fiber Composites, Integration, Fragmentation, Sensor

\section{INTRODUCTION}

Due to excellent electrical-mechanical coupling piezoelectric materials based on Lead-Zirconate-Titanate (PZT) are excellent candidates for use in adaptive material systems. Traditional PZT devices are composed of a PZT wafer with electrodes on the top and bottom as in the Rainbow [1] and Thunder [2] actuators. However, these wafer based devices suffer from low damage tolerance and actuation performance. Improvement on these limitations was found in the work of Bent and Hagood [3] at the Massachusetts Institute of Technology in the original development of the Active Fiber Composite (AFC) actuator system. AFC are composed of PZT fibers embedded in an epoxy matrix and sandwiched between two interdigitated electrodes (IDE). The AFC design surpasses the traditional PZT wafer in many areas. Due to their composite design AFC are more flexible than traditional PZT wafers and they are polarized along the fiber length, as opposed to polarization through the wafer thickness, and thereby utilize the piezoelectric properties in the $d_{33}$ direction, offering greater performance than wafers, which use the $d_{31}$ component. Different fiber arrangements and IDE patterns allow AFC to be tailored for specific applications more so than wafer devices.

In the realm of smart materials design $\mathrm{AFC}$ are interesting because they can be designed for specific sensor and actuator applications. Many applications can be imagined for AFC such as health monitoring, actuation, and vibration damping. However, few studies have addressed the characterization and performance limits of AFC or PZT wafer devices as a component in an adaptable structure. Numerous studies have addressed the ferroelastic and ferroelectric

\footnotetext{
" Corresponding author. Tel.: +41-44-823-4307; fax: +41-44-823-4496. e-mail: mark.melnykowycz@empa.ch
} 
properties of bulk PZT $[4,5,6,7]$ which lends insight into the physical principles governing AFC but does not provide enough information to fully extrapolate AFC performance given their composite structure. From the practical side, $\mathrm{AFC}$ characterization work has been performed for actuation performance and the specific application of angle of twist actuation of a Boeing active material rotor [8]. However, a clear understanding of AFC behavior and performance in severe operating environments has not been presented. Understanding AFC performance is closely linked with that of bulk PZT.

An adaptable structure requires the integration of devices into a host material. Laminar material systems offer an ideal starting point given their flexibility in shape and manufacturing. In addition, laminar integration research is interesting given the number of applications that are based on laminar composites and are using smart material principles such as tennis rackets [9], adaptive skis [10], and rotor blades [11,12]. To date, most research dealing with piezoelectric sensor/actuator integration has revolved around PZT wafer integration, with little research directed towards AFC-type devices. Therefore, investigating integration issues of AFC into composite laminates is relevant for many sectors.

\section{$1.1 \quad$ Integration}

Integration may be defined as any technique used to incorporate a piezoelectric device into a structure or material. In a laminate composite the two most basic integration techniques include "simple insertion" and "cutout insertion" or "embedding." Simple insertion entails simply placing the device between the laminate layers, essentially sandwiching it in place. Embedding or cutout insertion involves removing laminate material, and then placing the device into the void so that the device becomes continuous with the surrounding structure or material.

Crawley and Luis [13] investigated the integration of PZT wafer actuators into woven composites using analytical and experimental methods. In addition, they laid down design criteria for PZT actuators such as a high modulus of elasticity to allow stress transfer with the surrounding laminate. In static tension, the embedding of PZT devices were found to have little effect on the elastic modulus, but a $20 \%$ decrease in the ultimate tensile strength of glass/epoxy laminates embedded with PZT devices was observed. The question of electrical insulation in carbon/epoxy embedded composites was also addressed.

Vizzini et al. [14,15] studied the effects of layering technique on the stress transfer between embedded PZT devices and unidirectional composite layers. In a first study [14] an optimal interlacing design was proposed using finite element techniques. The second study [15] tested the design by placing glass slides in uni-directional (UD) graphiteepoxy laminates and performing tensile testing. The need for interlacing is not clear though. Paget et al. [16] performed tensile testing of carbon/epoxy laminates with simply inserted PZT wafers and only saw a $3 \%$ difference in mechanical properties between the laminate with integrated PZT wafers and the reference laminate. In addition, multiple studies $[17,18,19,20]$ suggest that with quasi-isotropic carbon-epoxy composite lay ups the integration technique does not greatly affect laminate strength. Mall et al. [17] showed a difference of $4 \%$ between the mechanical properties of composite laminates without integrated devices and those that employed the simple and cutout insertion techniques.

\subsection{Objectives}

The current study investigated integration of $\mathrm{AFC}$ in orthotropic woven glass/epoxy laminates. In addition, the effect of AFC integration on the mechanical integrity of the glass laminates was assessed as well as the effect of integration on AFC performance parameters. More specifically, the aim was to analyze the effect that AFC integration imposes on laminate integrity and the effect that integration has on AFC performance. Due to electro-mechanical coupling in tests using the AFC sensing properties will also lend great insight into the actuation performance given the same mechanical environment. To fulfill these aims, procedures and processes required for integrating AFC devices into glass fiber/epoxy laminates were developed. 


\subsection{Materials}

Glass fiber epoxy laminates were produced with a vacuum bagging technique using Isopreg HR 320P-40 plain weave pre-impregnated (pre-preg) composite plies supplied by Isolvolta (www.isovolta.com). AFC were produced using 250 $\mu \mathrm{m}$ diameter PZT-5A fibers produced by Smart Material Corp., Osprey Fl, USA (www.smart-material.com). The fibers were laminated with an epoxy system (Aradur 2954 hardener with Araldite LY564 resin) supplied by Vantico AG, Basel, Switzerland (www.vantico.com) and sandwiched between two sheets of $25 \mu \mathrm{m}$ thick Dupont Kapton 100 HN film (www.dupont.com) with screen-printed IDE. Electrical connections for the AFC were made using $250 \mu \mathrm{m}$ POLYSOL-180 wires supplied by Elektro-Feindraht AG. Dummy AFC (DAFC) were fabricated using Stesatape EP121-GR101/228-30 uni-directional (UD) glass pre-preg plies from Stesalit (www.stesalit.com) sandwiched between two sheets of $25 \mu \mathrm{m}$ thick $100 \mathrm{HN}$ polyimide Kapton film.

\subsection{Laminate manufacture}

Laminates composed of plain weave glass plies arranged in orthotropic stacking sequences were manufactured in a hot press using a vacuum bagging and pressing process. Plies were prepared by cutting sections of the pre-preg strips to dimensions of $30 \times 30 \mathrm{~cm}$. An aluminum tool plate was pre-heated to $80^{\circ} \mathrm{C}$ and placed on a waist level cantilever stand for proper arrangement of the plies. Pre-preg plies were arranged on the hot tool plate in a symmetric orthotropic manner. The tool plate was then placed in a press (pre-heated to $80^{\circ} \mathrm{C}$ ) and vacuum was applied via an external pump. The press was closed and the temperature was raised to $120^{\circ} \mathrm{C}$. When the temperature of the press reached $120^{\circ} \mathrm{C}$ a pressure of 5 bar was applied to the tool plate. According to manufacturer specifications [21] the resin system would fully cure after six minutes at $120^{\circ} \mathrm{C}$. The plate was removed from the press and the vacuum bag after 30 minutes to ensure a full cure cycle of the laminate.

\subsection{Active fiber composite and dummies manufacture}

AFC were manufactured by hand using $250 \mu \mathrm{m}$ diameter piezoelectric fibers and epoxy resin. The fibers were arranged, secured with tape and then the fiber layer was transferred to an aluminum tool plate with a Kapton film printed IDE. The epoxy was prepared with a ratio mass composition of 3:1 epoxy to hardener. Epoxy was applied to the fiber layer and the tool plate was degassed at $60^{\circ} \mathrm{C}$ to remove air from the matrix. Wires were connected to the electrodes using a conductive silver epoxy. A second Kapton/IDE layer was laid on top and a pressure was applied for 1 hour at $120^{\circ} \mathrm{C}$. The AFC were post cured for 8 hours at $160^{\circ} \mathrm{C}$ to ensure full cure. Three different types of AFC were manufactured for tensile testing (dimensions $2 \times 15 \mathrm{~cm}$ ). The first set included three AFC composed of only Kapton and PZT fibers with the epoxy matrix. The second set included three AFC with screen-printed IDE. Finally the last set consisted of three AFC with IDE, and were poled for 20 minutes with an applied voltage of $2.5 \mathrm{kV}$ at $80^{\circ} \mathrm{C}$. Eight AFC (dimension $3.3 \times 4.0 \mathrm{~cm}$ ) were also produced for integration into the GFRP laminates.

In order to optimize testing procedures, reduce manufacturing time and predict the effect AFC integration would have on the integrity of the laminates, DAFC were produced." A regime of tests, were carried out with DAFC in order to reduce costs and determine fracture behavior that could be expected with AFC. In order to simulate the AFC, DAFC were manufactured using Kapton film and UD glass pre-preg plies. The UD plies were arranged at a $+/-20^{\circ}$ orientation, and effectively matched the longitudinal modulus of the AFC. Kapton film was used in the DAFC to simulate the bonding characteristics with the laminate structure. The temperature was raised to $120^{\circ} \mathrm{C}$ and maintained for 90 minutes in accordance with the recommended curing cycle [22]. Nine DAFC were produced for tensile testing. DAFC for integration in the GFRP laminates had dimensions of $4 \times 4 \mathrm{~cm}$. 
AFC and DAFC were placed in different ply positions to ascertain the effect that varying device placement would have on tensile strength and delamination characteristics of the laminates. The elements were placed either "center," "off-center," or "far off-center" with respect to the middle of the ply cross-section. The term "center" refers to the center placement of AFC and DAFC with regard to the thickness of the final laminates (i.e., a position at the neutral axis of the laminate). Laminates utilizing simple insertion were composed of 8 plies and those used for cutout insertion were composed of 9 plies. This was done to maintain a comparable position from the neutral axis in the laminates for both integration methods.

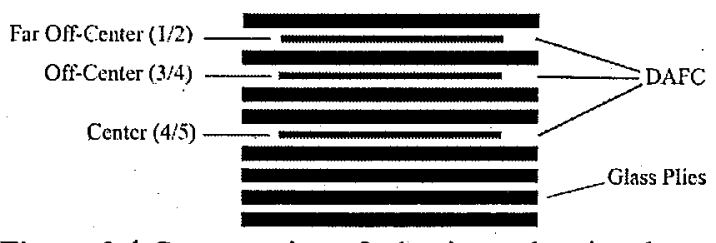

Figure 2.1 Cross-section of a laminate showing the placement positions of devices integrated via simple insertion.

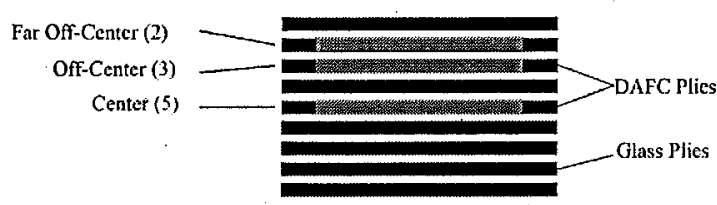

Figure 2.2 Cross-section of a laminate showing the placement positions of devices integrated via cutout insertion.

AFC and DAFC were integrated using either the simple (Fig. 2.1) or cutout insertion (Fig. 2.2) technique. An outline of element placement was mapped out on a ply using a permanent pen and drafting tools. For simple insertion the elements were placed inside their outline on the ply and successive plies were laid down as needed to complete the lay-up. In the cutout insertion technique sections corresponding to the size of the insert were cutout and removed from the pre-preg ply. The latter ply was laid down and AFC or DAFC were carefully placed in the cutout cavity. A schematic of AFC and the layout of plies and wire connections is shown in Fig 2.3. Protective layers of Teflon sheet and Mylar film with double-sided tape was placed on the edge of the integration ply to protect the wire connections from resin flow during processing.

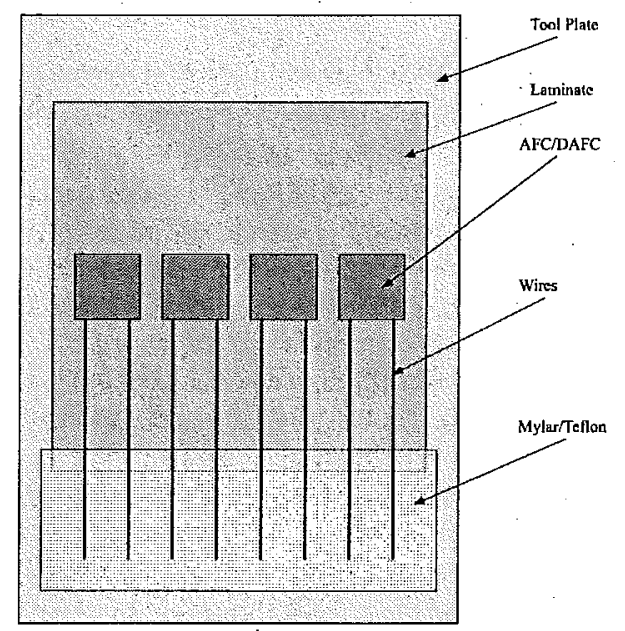

Figure 2.3 Schematic of a laminate with AFC/DAFC arranged and wires with Teflon/Mylar protection.

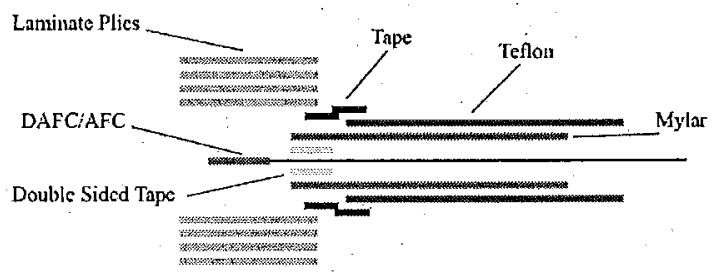

Figure 2.4 Schematic side view of Teflon/Mylar wire protection.

For DAFC integration two wires approximately $35 \mathrm{~cm}$ long were placed near the edge of each DAFC and extended to the Teflon/Mylar layer and secured using tape. A second layer of Teflon/Mylar was placed on top of the wire and pressed down. A schematic representation of the wire protection is shown in Fig 2.4. On both pieces of Mylar a layer of double-sided tape was placed on the edge that would touch the wires. This was done to create a barrier so that resin would not encase the wire connections during processing. 
Tensile testing was performed with an Instron 1251 test machine with four different test groups. Specific configurations are listed in Table 2.1. AFC and DAFC specimens were tested to determine their longitudinal mechanical properties. Reference laminate (containing no AFC/DAFC) and integrated (containing AFC/DAFC) laminate specimens were prepared in accordance with ASTM Standard D 3039/D 3039M-00. Both AFC/DAFC laminates were tested under the Laminate conditions in Table 2.1. For laminates with DAFC, five specimens were tested for each combination of integration procedure (cutout and simple insertion) and placement position (center, offcenter, far off-center) for a total of 30 specimens. In addition, five specimens were tested with two integrated DAFC in the far off-center position (far off-center symmetric). For laminates with AFC (only cutout insertion), eight total samples were tested (four with center placement and four with off-center placement). The mechanical oscillation (Lam. Cyclic) test was strain controlled and is described below in section 2.6. In each test group Aerolite epoxy glue was used to secure tab material to the ends of each specimen. Where specified, either a strain gage or an extensiometer ( $50 \mathrm{~mm}$ gage length) was used to determine strain during the test.

Table 2.1 Summary of tensile specimen dimensions and testing conditions.

\begin{tabular}{|lcccccc|}
\hline & Specimen Size & Tab Size & Strain Gage & Extensiometer & Load Cell & Load Rate \\
AFC & $2 \times 15(\mathrm{~cm})$ & $2 \times 2.5(\mathrm{~cm})$ & Yes & No & $10 \mathrm{kN}$ & $0.3 \mathrm{~mm} / \mathrm{min}$ \\
DAFC & $2 \times 25(\mathrm{~cm})$ & $2 \times 2.5(\mathrm{~cm})$ & No & Yes & $200 \mathrm{kN}$ & $2.0 \mathrm{~mm} / \mathrm{min}$ \\
Laminate & $30 \times 5(\mathrm{~cm})$ & $5 \times 5(\mathrm{~cm})$ & No & Yes & $200 \mathrm{kN}$ & $2.0 \mathrm{~mm} / \mathrm{min}$ \\
Lam. Cyclic & $30 \times 5(\mathrm{~cm})$ & $5 \times 5(\mathrm{~cm})$ & Yes & No & $200 \mathrm{kN}$ & $0.01 \% / \mathrm{sec}$ \\
\hline
\end{tabular}

\section{6}

\section{Mechanical oscillation test}

Performance of integrated AFC (IntAFC) was measured via a monotonic cyclic load test. AFC were integrated into the glass laminates using the cutout insertion technique. IntAFC were placed at the center and the off-center positions. The IntAFC test specimens had dimensions and test conditions as described in Table 2.1. A strain controlled test procedure was used to oscillate the specimens around a prescribed strain level at $10 \mathrm{~Hz}$ for one second with strain oscillation amplitude of $0.01 \%$ as shown in Fig 2.5 . Specimens were loaded twice to the ultimate strain for each test. Each specimen was loaded through the strain steps, up to the ultimate level and then taken back to zero strain before again loading to the same level. Successive tests were conducted with ultimate strain ranging from a minimum of $0.20 \%$ to a maximum of $0.70 \%$. A condensator with a capacity of $1277 \mathrm{nF}$ was connected in parallel to the AFC during the test. During testing the resulting signal from the IntAFC signal and strain data were collected via a data acquisition PC card and LabVIEW software as shown in Fig 2.6.

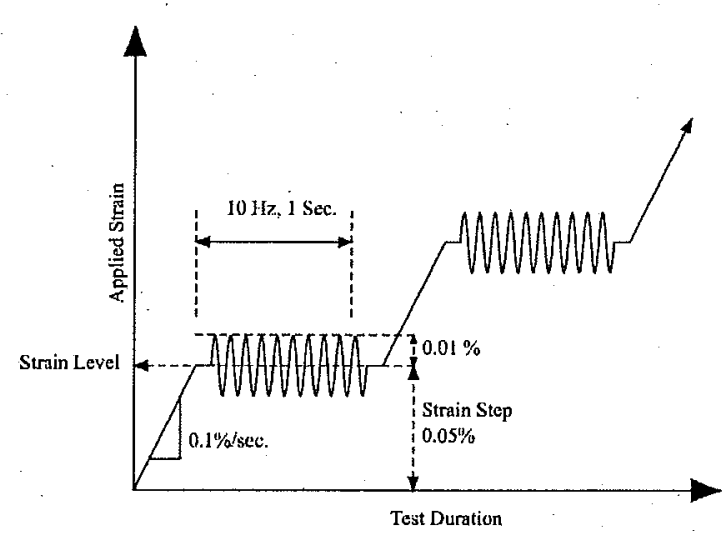

Figure 2.5 Applied strain program for mechanical oscillation cycle test.

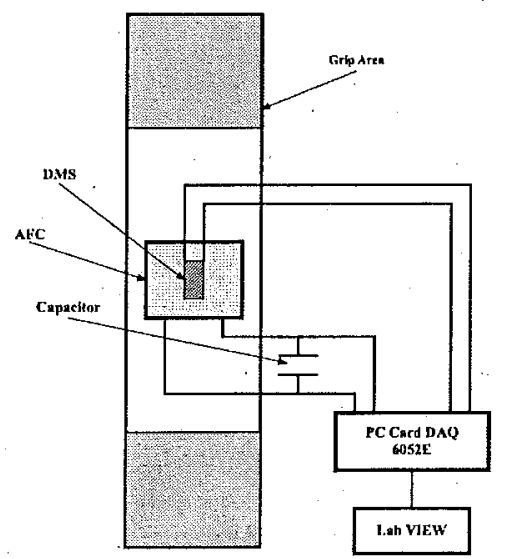

Figure 2.6 Experimental set-up for the monotonic cycle test of $30 \times 5 \mathrm{~cm}$ tensile specimens. 


\section{RESULTS \& DISCUSSION}

\subsection{AFC mechanical properties}

Fig 3.1 depicts the stress strain response of AFC specimens during tensile loading. The curves have been shifted to account for an initial loading region during testing, which is not thought to be representative of the true material behavior. The mechanical stress-strain response was highly nonlinear. The elastic modulus was calculated from the slope of the initial linear response between $0.01 \%-0.03 \%$. The average stress at failure $\left(\sigma_{\mathrm{MAX}}\right)$ was $47 \mathrm{MPa}$ with standard deviation of $8.2 \%$. A Young's Modulus (E) of $29 \mathrm{GPa}$ was found with a standard deviation of $4.7 \%$ and an ultimate strain $\left(\varepsilon_{\mathrm{MAX}}\right)$ of $0.25 \%$ with a high scatter of $16.8 \%$ standard deviation. Mechanical properties of the AFC and DAFC are given in Table 3.1 where the Young's Modulus values are in good agreement. The matching of the Young's Modulus between the AFC and DAFC allow integration tests using DAFC to be used to simulate the presence of an AFC.

Table 3.1 Results of AFC and DAFC tensile testing.

\begin{tabular}{|ccccccccc|}
\hline & \multicolumn{3}{c}{ AFC } & & & \multicolumn{3}{c}{ DAFC } \\
Avg. & $\sigma_{\text {MAX }}(\mathbf{M P a})$ & $\mathbf{E}(\mathbf{G P a})$ & $v$ & $\varepsilon_{\text {MAX }}(\%)$ & $\sigma_{\text {MAX }}(\mathbf{M P a})$ & $\mathbf{E}(\mathbf{G P a})$ & $\varepsilon_{\text {MAX }}(\%)$ \\
StDev & 43 & 29 & 0.38 & 0.25 & 537 & 31.38 & 1.86 \\
\%Dev & 3.49 & 1.36 & 0.02 & 0.04 & 52 & 1.10 & 0.11 \\
& 8.15 & 4.71 & 5.66 & 16.82 & 9.71 & 3.52 & 6.05 \\
\hline
\end{tabular}

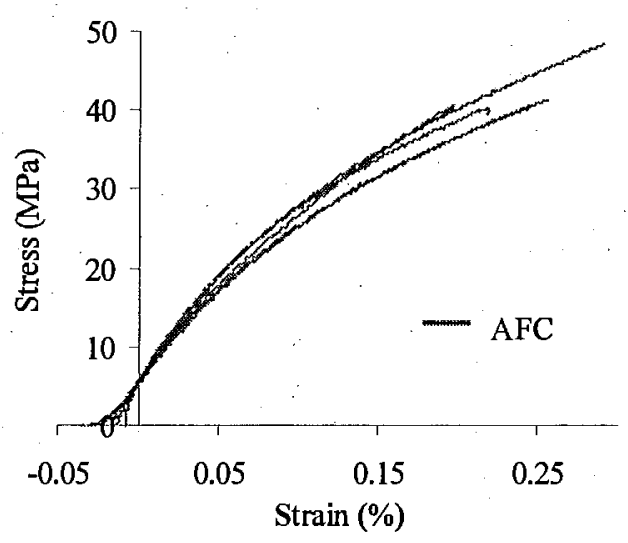

Figure 3.1 Stress strain behavior of AFC.

\subsection{Influence of integrated dummies/AFC on laminate tensile properties}

The stress/strain response of the glass laminates, with various configurations and standard deviation error bars are graphically represented in Figs 3.2, 3.3. As can be seen in Fig $3.2 \sigma_{\mathrm{MAX}}$ decreased with the integration of DAFC in the 
laminate and decreased further as the DAFC were placed far from the symmetry axis. Center placement decreased the $\sigma_{\text {MAX }}$ value by about $13 \%$ while the off-center and far off-center placements showed a drop in strength of $20 \%$ and $30 \%$ respectively. Although a quantifiable difference is seen due to integration, the type of integration technique (i.e. cutout vs. insertion) appeared to have little effect on the $\sigma_{\text {MAX }}$ values. Indeed, the difference between $\sigma_{\text {MAX }}$ values for the simple and cutout insertion specimens for each placement method were within the standard deviations. Similar behavior was seen for the $\varepsilon_{\mathrm{MAX}}$ values as shown in Fig 3.3.

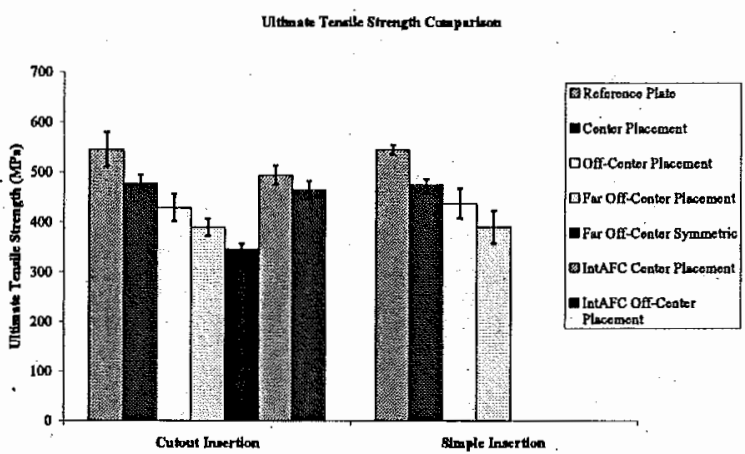

Figure 3.2 Ultimate tensile strength results for the various integration methods.

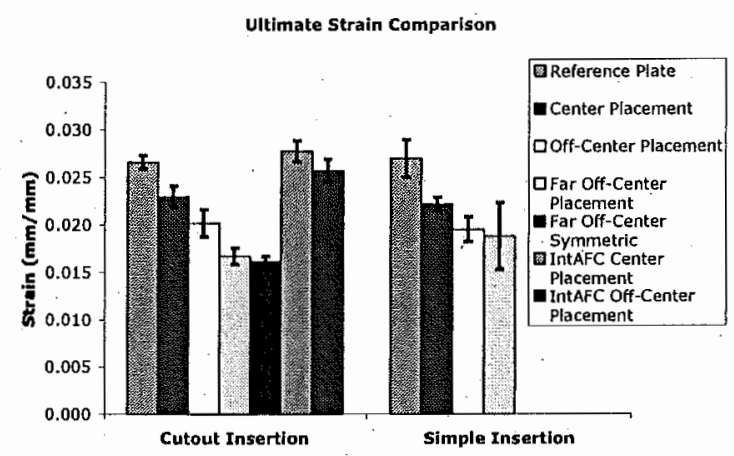

Figure 3.3 Ultimate strain results for the various integration methods.

In Fig 3.3 the center placement with simple insertion ultimate tensile strain decreased by nearly $18 \%$ and almost $14 \%$ for the cutout specimens. In the off-center case the ultimate tensile strains decreased by $24 \%$ and $28 \%$ respectively. These differences closely mimic the $\sigma_{\text {MAX }}$ behavior. In contrast to the $\sigma_{\text {MAX }}$ and $\varepsilon_{\text {MAX }}$ results, the Young's modulus data showed no significant difference for the different placement methods.

The stress/strain curves of the different laminates are displayed in Figs 3.4 and 3.5 for each insertion technique. Interestingly, the off-center specimens exhibited an extended strain region past their initial $\sigma_{\text {MAx }}$ values with both simple and cutout insertion techniques. This "yield point" corresponded with visual observations during the test of the onset of internal damage and delamination in the tensile specimens. After the "yield point" this extended region corresponded to extensive delamination prior to failure in the specimens. The laminates eventually failed via catastrophic transverse crack propagation. As can be seen from the two curves, the stress decreased from the initial yield point, reached an intermittent minimum, and rose to a second stress level where failure occurred. However, the two stress levels do not differ significantly. This behavior differed considerably from the center placement fracture behavior with DAFC inserted in the symmetric axis of the laminates where no such yield point was observed.

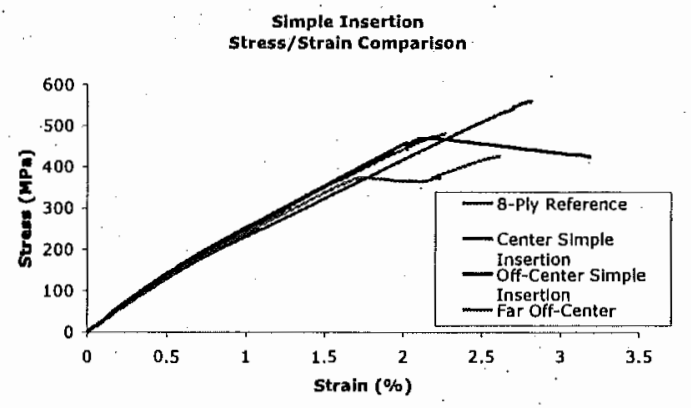

Figure 3.4 Stress/strain behavior for different placement methods using simple insertion.



Figure 3.5 Stress/strain behavior for different placement methods using cutout insertion. 
Using cutout insertion, the specimens with IntAFC displayed strain behavior similar to the ones with IntDAFC as seen in Fig 3.6. The failure of the samples in both cases occurred at similar stress levels. The center placement specimens displayed a smooth loading profile and fractured with comparable maximum stresses. It was seen previously that the off-center IntAFC had a lower $\sigma_{\mathrm{MAX}}$ value than the IntDAFC. However in both cases a yield point is evident before final failure. A difference is also seen in the Young's modulus data. The initial moduli are similar at low strain (below $0.03 \%$ ), above this limit the modulus of the samples with integrated AFC is lower than that of the samples with DAFC. The AFC tensile specimens had an ultimate tensile strain of $0.25 \%$. So, from a materials standpoint, beyond $0.25 \%$ damage may have ensued. If cracks developed in the IntAFC the stiffness of the structure would logically decrease. As seen in Fig 3.5 the initial slope appears similar, but changes beyond $0.50 \%$, possibly indicating damage in the AFC.

\subsection{Laminate Fracture Behavior}

Failure in the laminates with integrated dummies occurred via damage in the Kapton layer and subsequent transverse failure in the laminate. In the off-center IntDAFC delamination initiated at the DAFC/Glass interface and spread over the surface of the DAFC. Ultimate failure generally occurred in the glass plies directly at a horizontal edge of the DAFC and post-failure inspection of the samples showed that failure occurred in the Kapton layer. Part of the Kapton remained adhered to the DAFC core and part remained with the adjacent glass ply. Prior to full laminate failure the DAFC essentially delaminated within the glass plies without complete failure to the specimen. Characteristic IntDAFC failure specimens are shown in Fig 3.6 where failure occurred near or on the horizontal edge (normal to the loading direction) of the DAFC.

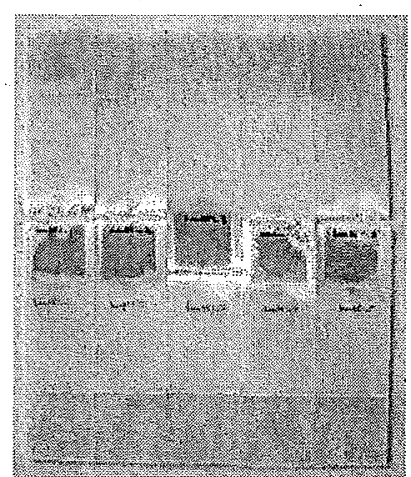

Figure 3.6 Far off-center symmetric cutout insertion specimens with DAFC.

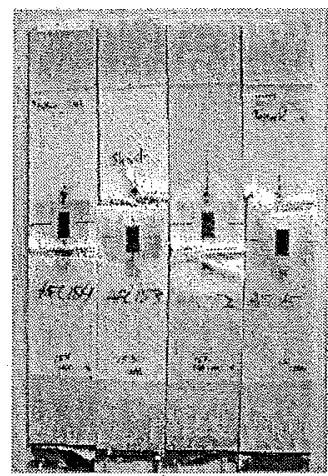

Figure 3.7 Center placement symmetric cutout insertion specimens with AFC.

Characteristic IntAFC specimens, displayed in Fig 3.7 exhibited fracture behavior slightly different from that of the IntDAFC. The samples with center IntAFC contained fractures near the horizontal edge, as was the case with the IntDAFC. However, in some cases, an additional vertically oriented fracture above the AFC appeared, but most specimens failed similar to the IntDAFC laminates. Off-center IntDAFC failed almost universally near the DAFC edge. However, with the off-center IntAFC the transverse ultimate failures resided past the AFC edge, more inside the planar area of the AFC. In these cases the fracture lines passed through the Kapton layer and more towards the AFC center. This Kapton failure did not coincide with the DAFC behavior. Rather, the Kapton delaminated from the PZT fiber layer and remained coincident with the laminate until failure. All of the IntAFC specimens had delamination in the glass plies, resulting from failure of the AFC. This delamination occurred after ultimate failure and is indicated as the white regions on the specimens, contrasting with the off-white color of the laminates near the tabbed ends.

\subsection{Integrated DAFC and AFC Fracture Behavior}

Differences were seen in the fracture behavior between the laminate with center and off-centered IntDAFC. Although the fracture locations were similar (transverse failure along the horizontal edge) the damage in the Kapton layer suggests two different types of failure. Fig 3.8 displays images of a center and off-center DAFC post failure. Even at 
the macroscopic scale the two specimens show different failure characteristics. The center IntDAFC (Fig 3.8a) has a largely uniform surface consisting of a dimpled pattern, except at the horizontal edge where irregular ripping or shearing is seen. By comparison the off-center IntDAFC (Fig 3.8b) has a non-uniform character with large areas where the Kapton has ripped away from the DAFC core material.

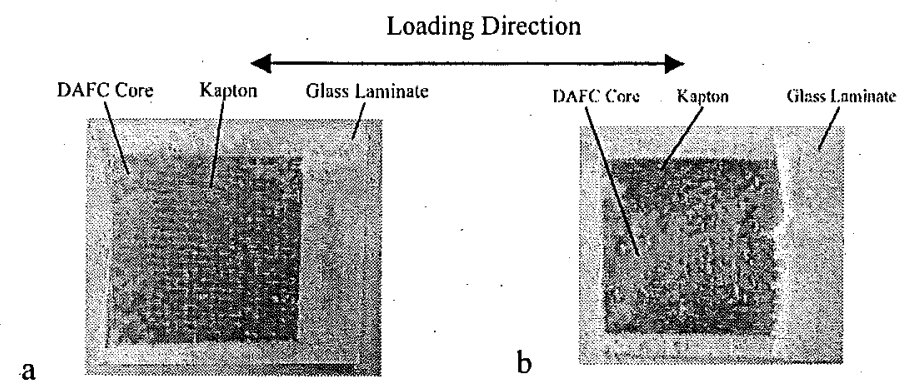

Figure 3.8 Center (a) and off-center (b) placement DAFC post failure.

The discontinuous failure nature of the off-center IntDAFC Kapton layer suggests a non-uniform stress state. The non-uniform failure can be characterized as ripping, suggesting failure due to shearing forces. It is also pertinent to note that the Kapton near the side edges of the DAFC (parallel to the tensile loading direction) do not show the same discontinuous ripping character. These side regions are largely undamaged. By comparison, both the off center and center specimens retain the discontinuous or ripping failure modes near the horizontal edge (perpendicular to the loading direction). During tensile testing it was observed that visible failure initiated at the horizontal edge of the $\mathrm{DAFC}$ and then propagated until failure: The ripping failure at the horizontal edge is indicative of the initial failure observed during testing and suggests failure in the laminates occurred due to shear forces at the horizontal DAFC edge. The uniformity of the center placement specimen implies that subsequent failure was influenced by normal stresses at the Kapton/laminate interface. Conversely, shear loading appears to have dominated the failure behavior in the off-center specimen as evidenced by the ripping patterns. The center and off-center IntAFC are shown post failure in Fig 3.9.

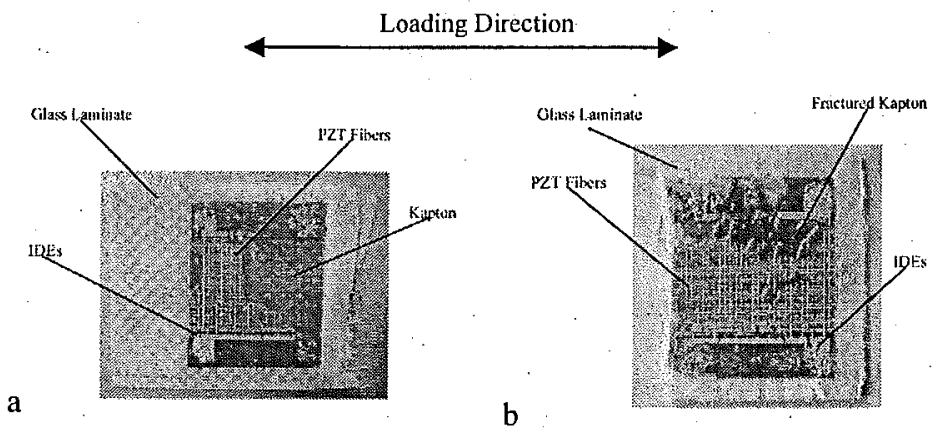

Figure 3.9 Off-center (a) and center (b) placement AFC post failure.

The IntAFC showed some fracture characteristics coincident with the IntDAFC. In most cases fractures occurred at the horizontal edge or separation occurred between the insert and surrounding laminates such that one face of the insert remained adhered to the adjacent laminate and the other face delaminated. Similarly, in the IntAFC the IDE remained adhered to the PZT fibers, while the Kapton pulled away with the adjacent ply. However, the Kapton in the center IntAFC shows signs of shear failures, without the dimple character reminiscent of the center IntDAFC. This discrepancy can be attributed to the fundamental differences in the AFC and DAFC core material. The PZT fibers offer a very different surface texture than the DAFC core (UD glass), which is a planar, therefore making a direct comparison questionable. 


\subsection{PZT Fiber Fracture}

The most significant difference between the IntDAFC and IntAFC behavior concerned their internal failure characteristics. The IntDAFC showed failure in the Kapton layer, while the DAFC core material always remained intact, as would be expect given its higher failure strength. Fracture within the IntAFC included Kapton delamination as well as fracturing of the PZT fibers. Fig 3.10 shows an IntAFC post failure. The fracture lines traversed horizontally through the fibers and surrounding matrix, characteristic of brittle tensile failure in fiber/matrix composites. In Fig 3.10 failure in the PZT fibers is evidenced by the shifting of the fiber outline in conjunction with the crack line, indicating that the PZT fibers have fragmented and formed free surfaces.

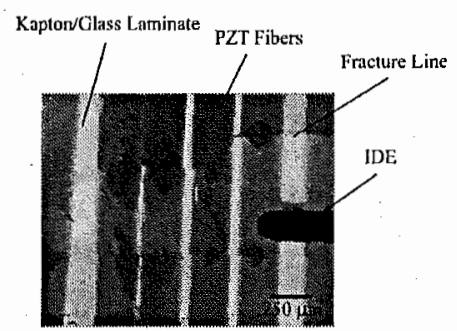

Figure 3.10 Fracture behavior through PZT fibers and AFC matrix in off-centered AFC.

\subsection{Influence of mechanical strain on the performances of AFC as sensors}

AFC strain sensitivity performance in response to the cyclic strain test is shown in Fig 3.11 where sensitivity is defined as the amplitude of the AFC signal response during oscillation. When loaded from $0.05 \%$ up to $0.70 \%(0.70 \%$ $1^{\text {st }}$ Run in Fig 3.3) AFC sensitivity shows stability in the strain range of $0.05 \%-0.20 \%$. A severe slope change is seen when the applied strain reaches $0.25 \%$. Beyond that level sensitivity decreases in a near-linear manner until a second slope change at $0.45 \%$. Beyond $0.45 \%$ sensitivity continues to decline, but at a much reduced rate.

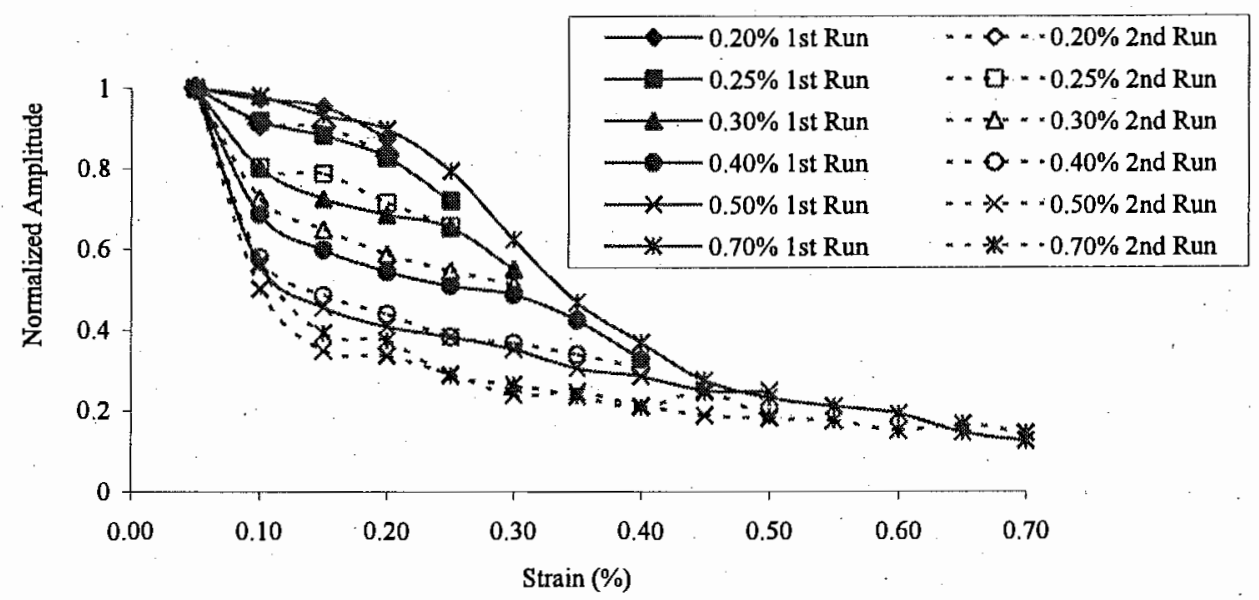

Figure 3.11 Amplitude response of AFC at successive strain levels. 
When loaded a second time to $0.70 \%\left(0.70 \% 2^{\text {nd }}\right.$ Run $)$ sensitivity drops off dramatically between $0.05 \%-0.10 \%$. It then approaches the plateau character exhibited in the first loading of beyond $0.45 \%$. Intermediate load curves show sensitivity behavior up to the strains of $0.20 \%, 0.25 \%, 0.30 \%, 0.40 \%$, and $0.50 \%$. Throughout these intermediate curves it is evident that the $2^{\text {nd }}$ cycle of each strain step exhibits quite similar behavior of the $1^{\text {st }}$ cycle of each higher subsequent strain step. For example, the $0.20 \% 2^{\text {nd }}$ run exhibits the same sensitivity as the $0.25 \% 1^{\text {st }}$ run and the $0.25 \% 2^{\text {nd }}$ run matches the $0.35 \% 1^{\text {st }}$ run and so on. For the intermediate strain curves a slope change is seen at the end of each curve. The most critical slope changes are seen at $0.20 \%$ and $0.45 \%$ with a near-linear signal degradation path existing between $0.20 \%$ and $0.45 \%$ strain. This slope change disappears in strains beyond $0.45 \%$. The strain signal amplitude drops off severely beyond $0.20 \%$, approaches a minimum after $0.50 \%$, and remains constant through $0.70 \%$. When loaded beyond $0.45 \%$ AFC sensitivity curve retains an inverse nature, dropping from $0.05 \%$ and approaching a minimum around $0.30 \%$ strain. One of the most interesting findings however, is that performance recovers to the maximum level at low strains. Despite sensitivity degradation at higher strains, when strained to $0.05 \%$ all specimens exhibited high sensitivity. In the case of fiber breakage, a disruption in the electrical field paths in the PZT would also hinder AFC performance. Reducing strains in the AFC would allow cracks in the fiber layer to close, explaining the performance recovery.

\section{CONCLUSION}

In the present study AFC sensor/actuators were integrated into glass/epoxy laminates to examine how their presence affected laminate integrity and to investigate IntAFC performance. Laminates integrated with AFC and DAFC were tensile tested to ascertain the effect of DAFC/AFC integration on laminate integrity. DAFC were used to simulate the effect of an integrated $A F C$, on laminate integrity when placed at different distances from the symmetric axis of the laminate and AFC were found to affect laminate integrity comparably with the DAFC. As AFC/DAFC were placed away from the symmetric axis of the laminate ultimate strength and strain decreased in a linear fashion while Young's modulus showed little change. The laminate strength dropped by $12 \%, 21 \%$, and $29 \%$ as DAFC were placed, respectively; in the center, off-center and far off-center positions. The corresponding standard deviation values were $4 \%, 7 \%$, and $5 \%$. In close agreement with AFC laminates, strength dropped by $10 \%$ and $15 \%$ for the center and offcenter placements with standard deviations of $4 \%$ in both cases. The strength reduction is attributed to the presence of a non-uniform stress state through the laminate thickness leading to a linear reduction in mechanical properties as the DAFC/AFC position approached the laminate surface. The essential difference between AFC and DAFC behavior resided in their specific failure mechanisms. DAFC failed via Kapton failure and delamination. Conversely, AFC failure also included the strength reduction related to PZT fiber fragmentation. Despite these differences both exhibited similar behavior when integrated into GFRP. laminates. Almost universally ultimate failure in the laminates occurred at the horizontal edge of the inclusion and then propagated through the material. In essence, failure in the laminate did not differentiate between the presence of an AFC or DAFC. Therefore, the DAFC testing scheme can be used as a tool to ascertain the effect of AFC integration in mechanical environments. In addition, the characterization of AFC elements integrated in GFRP laminates in a tensile strain environment was investigated. AFC performance was found to be stable up to a strain of $0.20 \%$. A degradation behavior is seen between $0.20 \%-0.40 \%$ due to fragmentation in the PZT fiber layer which saturated beyond $0.45 \%$ strain. However, performance is recovered at lower strains, and is attributed to crack closure in the PZT fiber layer.

\section{ACKNOWLEDGMENTS}

Funding for this work was provided by Empa in connection with the Adaptive Material Systems initiative. In addition the author would like to thank colleagues in the Institute for Mechanical Systems (IMES) at the Swiss Federal Institute of Technology (ETH) in Zurich, Switzerland for beneficial information exchange.

\section{REFERENCES}

[1] Haertling GH 1994 RAINBOW Ceramics - A new type of ultra-high-displacement actuator Amer. Ceram. Soc. Bull. 73 93-96 
[2] Mossi KM, Selby GV, Bryant RG 1998 Thin-layer composite unimorph ferroelectric driver and sensor properties Mater. Lett. 35 39-49

[3] Bent AA, Hagood NW 1997 Piezoelectric fiber composites with interdigitated electrodes J. Int. Mat. Syst. Struct. 8 903-919

[4] Lynch CS The effect of uniaxial stress on the electro-mechanical response of 8/65/35 PLZT 1996 Acta Mater. 44

4137-4148

[5] Cao H, Evans AG 1993 Nonlinear deformation of ferroelectric ceramics J. Am. Ceram. Soc. 76 890-896

[6] Tanimoto T, Yamamoto K, Morii T 1995 Nonlinear stress-strain behavior of piezoelectric ceramics under tensile loading Proc. 9th IEEE International Symp. on Applications of Ferroelectrics

[7] Schäufele AB, Härdtl KH 1996 Ferroelastic properties of lead zirconate titanate ceramics J. Am. Ceram. Soc. 79 $2637-2640$

[8] Wickramasinghe VK, Hagood NW 2004 Materials characterization of active fiber composites for integral twistactuated rotor blade application Smart. Mater. Struct. 13 1155-1165

[9] Head inc. Active Electronic Dampening System (EDS) Protector Racquet www.head.com/tennis/

[10] Head inc. Intelligence ${ }^{\mathrm{TM}}$ Ski System www.head/ski.com

[11] Hall SR, Prechtl EF 1996 Development of a piezoelectric servoflap for helicopter rotor control Smart Mater. Struct. 5 26-34

[12] Giurgiutiu V 2000 review of Smart-Materials Actuation Solutions for Aeroelastic and Vibration Control $J$. Intelligent Mater. Sys. and Struct. 11 525-544

[13] Crawley EF, de Luis, J 1987 Use of piezoelectric actuators as elements of intelligent structures AIAA 25 13731385

[14] Vizzini AJ, Xingh DA 1994 Structural integrity of composite laminates with interlaced actuators Smart Mater. Struct. $671-79$

[15] Vizzini AJ, Shukla DR 1996 Interlacing for improved performance of laminates with embedded devices Smart Mater. Struct. 5 225-229

[16] Paget CA, Levin K 1999 Structural integrity of composites with embedded piezoelectric ceramic transducer $S P I E$ Proc. 3668 306-313

[17] Mall S, Coleman JM 1998 Monotonic and fatigue loading behavior of quasi-isotropic graphite/epoxy laminate embeddd with piezoelectric sensor Smart Mater. Struct. 7 822-832

[18] Mall S, Hsu TL 2000 Electromechanical fatigue behavior of graphite/epoxy laminate embedded with piezoelectric actuator Smart Mater. Struct. 978-84

[19] Mall S 2002 Integrity of graphite/epoxy laminate embedded with piezoelectric sensor/actuator under monotonic and fatigue loads Smart Mater. Struct. 11 527-533

[20] Yocum M, Abramovich G, Grunwald A, Mall S 2003 Fully reversed electromechanical fatigue behavior of composite laminate with embedded piezoelectric actuator/sensor Smart Mater. Struct. 12 556-564

[21] Isopreg HR 320P-40 Technical Data Sheet, Isolvolta Prepregs.

[22] Stesatape EP121-GR101/228-30 Product Data Sheet. Stesalit Inc, www.stesalit.com. 